\title{
THE SOLUTIONS TO TWO PROBLEMS ON PERMUTATIONAL PRODUCTS
}

\author{
R. B. J. T. ALLENBY \\ (Received 13 May 1980; revised 14 October 1980) \\ Communicated by D. E. Taylor
}

\begin{abstract}
The two problems, both raised in the literature, are: (I) Is there, amongst all the permutational products (p.p.s) on the amalgam $\mathfrak{A}=(A, B ; H)$ at least one which is a minimal generalized regular product? (II) If one of the p.p.s on $\mathfrak{A}$ is isomorphic to the generalized free product (g.f.p.) $F$ on $\mathscr{A}$ are they all? We answer both of them negatively.
\end{abstract}

1980 Mathematics subject classification (Amer. Math. Soc.): 20 E 22.

Keywords: generalized free product, generalized regular product, free nilpotent group, permutational product, free group.

\section{Preliminaries}

Let $F$ be the g.f.p. on the amalgam $\mathfrak{A}=(A, B ; H)$ and let $G$ be any group generated by $\mathfrak{A}$. Let $\phi$ denote the homomorphism from $F$ onto $G$ which extends the identity maps on $A$ and $B$. Then $G$ is called a generalized regular product (Wiegold (1961)) on $\mathfrak{A}$ if and only if ker $\phi \subseteq[A, B]$, the cartesian subgroup of $F$. It was proved in Allenby (1969) that every p.p. is a generalized regular product. Further it is clear from a Zorn's Lemma argument that there exist in $F$ normal subgroups $N$ maximal with respect to both (i) $N \subseteq[A, B]$ and (ii) ( $a \in A, b \in$ $B$ and $\left.a b^{-1} \in N\right) \rightarrow a=b \in H$. Then, for such an $N$, the group $F / N$ is a minimal generalized regular product on $\mathfrak{A}$. Thus minimal generalized regular products exist on each amalgam. Gregorac (1969) asked if at least one of them was a p.p. on $\mathfrak{A}$.

○ Copyright Australian Mathematical Society 1981 
Assuming the reader to be familiar with the p.p. construction (see B. $\mathbf{H}$. Neumann (1960)) problem (II) is self explanatory. It arose (Allenby and Tang (1975)) in connection with investigations into the Frattini subgroups of g.f.p.s.

\section{The examples}

(I) We take $A=\langle a, b ;[a, b, a]=[a, b, b]=1\rangle$, the free second nilpotent group of rank $2, B=\langle c:\rangle$, and $H=\left\langle c^{2}\right\rangle=\langle b\rangle$. Theorem 2 of B. $\mathrm{H}$. Neumann (1960) shows that since $H$ is central in $B$, the isomorphism type of each p.p. on $\mathfrak{A}=(A, B ; H)$ is independent of the choice of transversal of $H$ in $A$. For each p.p. on $\mathfrak{U}$ we choose this transversal to be the set of elements $a^{i}[a, b\}^{j}$. As a transversal of $H$ in $B$ we choose $\left\{c^{2 m+1}, c^{2 n}\right\}$. By a theorem of Mal'cev (1949), $\mathfrak{A}$ is embeddable in a nilpotent group of class 2 . Consequently the generalized free second nilpotent product $W$, say, on $\mathfrak{A}$ exists (Wiegold (1959), Definition 4.1, Theorem 4.6). Further $W$ is a generalized regular product on $\mathfrak{A}$, and is isomorphic to $F /[A, B, F]$ (Wiegold 1959, Theorem 4.6). Thus, to solve problem (I) we only need show that the kernel of the natural map $\phi_{P}$ from $F$ onto each p.p. $P$ on $\mathfrak{A}$ lies properly inside $[A, B, F]$.

By Allenby (1969) ker $\phi_{P} \subseteq[A, B] \subseteq F$. Since $F$ is generated by the subgroups $\langle a\rangle$ and $\langle c\rangle$ we have $[A, B]=F^{\prime}=[\langle a\rangle,\langle c\rangle]$. Thus every element $X$ of $[A, B]$ is expressible in the form

$$
X=\prod_{i=1}^{t}\left[a^{A_{i}}, c^{2 B_{i}}\right]\left[a^{c_{i}}, c^{2 D_{i}+1}\right]\left[c^{2 F_{i}}, a^{E_{i}}\right]\left[c^{2 H_{i}+1}, a^{G_{i}}\right],
$$

where the $A_{i} B_{i}, \ldots, H_{i}$ are all integers, possibly 0 .

Now each p.p. $P$ on $\mathfrak{A}$ is a permutation group on a set of triples of the form $\left(a^{i}[a, b]^{j}, c^{2 m+1}, b^{k}\right)$ or $\left(a^{i}[a, b\}^{j}, c^{2 n}, b^{k}\right)$. The effect on these triples of the permutation

$$
\begin{aligned}
\rho(X)= & \prod_{i=1}^{t}\left[\rho\left(a^{A_{i}}\right), \rho\left(c^{2 B_{i}}\right)\right]\left[\rho\left(a^{C_{i}}\right), \rho\left(c^{2 D_{i}+1}\right)\right] \\
& \cdot\left[\rho\left(c^{2 F_{i}}\right), \rho\left(a^{E_{i}}\right)\right]\left[\rho\left(c^{2 H_{i}+1}\right), \rho\left(a^{G_{i}}\right)\right]
\end{aligned}
$$

is, on the one hand, the identity permutation (since $X \in \operatorname{ker} \phi_{P}$ ). On the other, one readily checks that

$$
\left(a^{i}[a, b]^{j}, c^{2 m+1}, b^{k}\right)^{\rho(X)}=\left(a^{i}[a, b]^{j+U}, c^{2 m+1}, b^{k}\right)
$$

whilst

$$
\left(a^{i}[a, b]^{j}, c^{2 n}, b^{k}\right)^{\rho(X)}=\left(a^{i}[a, b]^{j+V}, c^{2 n}, b^{k}\right),
$$


where

$$
U=\sum_{i=1}^{t} A_{i} B_{i}+C_{i} D_{i}-E_{i} F_{i}-G_{i} H_{i}+(m-n)\left(G_{i}-C_{i}\right)
$$

and

$$
V=\sum_{i=1}^{t} A_{i} B_{i}+C_{i} D_{i}-E_{i} F_{i}-G_{i} H_{i}+(n-m-1)\left(G_{i}-C_{i}\right) .
$$

Since $[a, b]$ has infinite order we have $U=V=0$. Hence $\Sigma_{i-1}^{t}\left(G_{i}-C_{i}\right)=0$ and $Z=\sum_{i=1}^{t}\left(A_{i} B_{i}+C_{i} D_{i}-E_{i} F_{i}-G_{i} H_{i}\right)=0$. Let $\bar{X}$ be the image of $X$ in $\bar{F}=F /[A, B, F]$. Since $[\bar{A}, \bar{B}]$ is central in $\bar{F}$ we have

$$
\bar{X}=\sum_{i=1}^{t}[\bar{a}, \bar{c}]^{2 A_{i} B_{i}}[\bar{a}, \bar{c}]^{C_{i}\left(2 D_{i}+1\right)}[\bar{c}, \bar{a}]^{2 F_{i} E_{i}}[\bar{c}, \bar{a}]^{\left(2 H_{i}+1\right) G_{i}}
$$

But then $\bar{X}=\overline{1}$ in $\bar{F}$-since $Z=\sum_{i=1}^{t}\left(G_{i}-C_{i}\right)=0$. Thus ker $\phi_{P} \subseteq[A, B, F]$.

Finally, this inequality is strict. For the permutation $[\rho(a), \rho(c), \ldots, \rho(c)]$, with $\alpha+2$ copies of $\rho(c)$, maps

$$
\left(a^{i}[a, b]^{j}, c^{2 m+1}, b^{k}\right) \text { to }\left(a^{i}[a, b]^{j+\gamma}, c^{2 m+1}, b^{k}\right),
$$

where $Y=(-1)^{\alpha} 2^{\alpha}(2 m-2 n+1)$. This suffices.

(II) Here we take $A=\langle a:\rangle \times H, B=H \times\langle b:\rangle$ where $H$ is free of countably infinite rank on the free generators

$$
\left\{h_{1}, h_{2}, \ldots, k_{1}, k_{2}, \ldots, l_{1}, l_{2}, \ldots, m_{1}, m_{2}, \ldots\right\} \text {. }
$$

For the first p.p., $P_{1}$, on $\mathfrak{A}=(A, B ; H)$ we choose $\left\{1, a, a^{-1}, a^{2}, a^{-2}, \ldots\right\}$, $\left\{1, b, b^{-1}, b^{2}, b^{-2}, \ldots\right\}$ as transversals for $A$ and $B$ respectively modulo $H$. It is easy to check that $P_{1}$ is isomorphic to $\langle a\rangle \times H \times\langle b\rangle$.

For the second p.p., $P_{2}$, we choose, as transversals for $A$ and $B$ respectively modulo $H$, the sets

$$
\left\{1, a h_{1}, a^{-1} k_{1}, a^{2} h_{2}, a^{-2} k_{2}, \ldots\right\} \text { and }\left\{1, b l_{1}, b^{-1} m_{1}, b^{2} l_{2}, b^{-2} m_{2}, \ldots\right\} \text {. }
$$

Recall that ker $\phi_{P_{2}}: F \rightarrow P_{2}$ is contained in $[A, B] \subseteq F$. We shall show that $\operatorname{ker} \phi_{P_{2}}$ is trivial. Thus let

$$
X=\prod_{i=1}^{t}\left[a^{\alpha_{i}} H(i), b^{\beta_{i}} K(i)\right]\left[b^{\delta_{i}} M(i), a^{\gamma_{i}} L(i)\right]
$$

be a typical element of $[A, B]$. Here the $H(i), K(i), L(i), M(i)$ are from $H$ and hence products of $h s, k s, l s, m s$ and their inverses. Since $a, b$ centralize $H, X$ can be re-written

$$
\begin{aligned}
X & =\prod_{i=1}^{t}\left[a^{\alpha_{i}}, b^{\beta_{i}}\right]\left[a^{\gamma_{i}}, b^{\delta_{i}}\right]^{-1} \cdot \prod_{i=1}^{t}[H(i), K(i)][L(i), M(i)]^{-1} \\
& =Y \cdot Z \text { say. }
\end{aligned}
$$


Suppose that the maximum suffix attached to any $h, k, l$ or $m$ occurring in the $H(i), K(i), L(i), M(i)(1 \leqslant i \leqslant t)$ is $v$. Set

$$
u=\max \left(\sum_{i=1}^{t}\left|\alpha_{i}\right|+\left|\gamma_{i}\right| \text { and } \sum_{i=1}^{l}\left|\beta_{i}\right|+\left|\delta_{i}\right|\right)
$$

and set $\lambda>2 \max \{u, v\}$.

Consider the effect of $\rho(X) \in P_{2}$ on $\left(a^{\lambda} h_{\lambda}, b^{\lambda} l_{\lambda}, 1\right)$. Now

$$
\begin{aligned}
\rho(X)= & \prod_{i=1}^{t}\left[\rho\left(a^{\alpha_{i}}\right), \rho\left(b^{\beta_{i}}\right)\right]\left[\rho\left(a^{\gamma_{i}}\right), \rho\left(b^{\delta_{i}}\right)\right]^{-1} \\
& \cdot \prod_{i=1}^{t}[\rho(H(i)), \rho(K(i))][\rho(L(i)), \rho(M(i))]^{-1} \\
= & \rho(Y) \cdot \rho(Z) .
\end{aligned}
$$

One can check, recalling that $\lambda>2 \max \{u, v\}$ and that $a$ and $b$ centralize $H$, that $\left(a^{\lambda} h_{\lambda}, b^{\lambda} l_{\lambda}, 1\right)^{\rho(Y)}=\left(a^{\lambda} h_{\lambda}, b^{\lambda} l_{\lambda}, U\right)$ where

$$
U^{-1}=\prod_{i=1}^{t}\left[h_{\lambda-\alpha_{i}}^{-1} h_{\lambda}, l_{\lambda-\beta_{i}}^{-1} l_{\lambda}\right]\left[h_{\lambda-\gamma_{i}}^{-1} h_{\lambda}, l_{\lambda-\alpha_{i}}^{-1} l_{\lambda}\right]^{-1} .
$$

(Notice that no $k_{i}$ nor $m_{i}$ appears in (*) precisely because $\lambda$ was chosen sufficiently large.) Now (*) is a product of commutators (and their inverses) each belonging to the natural free generating set for the derived group of the free subgroup of $\boldsymbol{H}$ generated by

$$
\left\{h_{\lambda}, k_{\lambda}\right\} \cup\left\{h_{\lambda-\alpha_{i}}^{-1} h_{\lambda}, h_{\lambda-\gamma_{i}}^{-1} h_{\lambda}, l_{\lambda-\beta_{i}}^{-1} l_{\lambda}, l_{\lambda-\delta_{i}}^{-1} l_{\lambda} \quad(1<i<t)\right\} .
$$

It follows that $U=1$ in $H$ if and only if $Y=1$ in $F$.

Finally, if $Y=1$, then $\rho(X)=\rho(Z)$ acts trivially on $\left(a^{\lambda} h_{\lambda}, b^{\lambda} l_{\lambda}, 1\right)$ if and only if $Z=1$. If $Y \neq 1$ then $\left(a^{\lambda} h_{\lambda}, b^{\lambda} l_{\lambda}, 1\right)^{\rho(X)}=\left(a^{\lambda} h_{\lambda}, b^{\lambda} l_{\lambda}, V\right)$ where $V=U^{-1} Z$. But $V \neq 1_{H}$ since, by choice of $\lambda$, the suffices occurring on the $h s$ and $l s$ in $U$ are disjoint from those occurring on the $h s, k s, l s$ and $m s$ in $Z$.

\section{References}

R. B. J. T. Allenby (1969), 'Permutational products and regular products of groups', Trans. Amer. Math. Soc. 136, 537-543.

R. B. J. T. Allenby and C. Y. Tang (1975), 'On the Frattini subgroups of generalized free products and the embedding of amalgams', Trans. Amer. Math. Soc. 203, 319-330.

R. J. Gregorac (1969), 'On permutational products of groups', J. Austral. Math. Soc. 10, 111-135.

A. I. Mal'cev (1949), 'Nilpotent torsion-free groups', Izv. Akad. Nauk SSSR Ser. Mat. 13, 201-212.

B. H. Neumann (1960), 'Permutational products of groups', J. Austral. Math. Soc. 1, 299-310. 
J. Wiegold (1959), Nilpotent products of groups with amalgamations', Publ. Math. Debrecen 6, 131-168.

J. Wiegold (1961), 'Some remarks on generalised products of groups with amalgamations', Math. Z. 75, 57-78.

\section{Department of Pure Mathematics}

University of Leeds

Leeds LS2 9JT, England 